\title{
A DESCRIPTIVE ANALYSIS OF THE INDICATIONS FOR CAESAREAN SECTION IN LUMBINI ZONAL HOSPITAL, BUTWAL.
}

\author{
Shreedhar Acharya ${ }^{1}$, Bhaktabatsal Raut ${ }^{1}$
}

\section{ABSTRACT}

AIM:

This study was done to find out the incidence and to analyse the various indications for caesarean sections at Lumbini Zonal Hospital.

\section{MATERIALS \& METHODS:}

This was a hospital based retrospective study done at Lumbini Zonal Hospital, Butwal. The case files of all the women who had caesarean delivery over the period of one year from 2071/4/1 to 2072/3/32 were reviewed. Various indications of caesarean sections were analysed for the incidence, age, parity, elective vs emergency caesareans.

\section{RESULTS:}

Out of 7589 deliveries, 1316 (17.34\%) had caesarean section. Most common indications were previous caesarean 275 (20.89\%), non progress of labour $245(18.61 \%)$, cephalopelvic disproportion $222(16.86 \%)$, fetal distress $215(16.33 \%)$, etc. Majority of caesarean $1226(93.16 \%)$ was done in the age group of 20-30yrs, primipara $702(53.34 \%)$, and elective caesarean sections $766(58.2 \%)$.

\section{CONCLUSION:}

Caesarean section rate at Lumbini Zonal Hospital was optimal. Most common indication was repeat caesarean. The overall reduction in caesarean section rate can be met through reduction of elective caesarean with the promotion of trial of labour.

KEYWORDS: Caesarean sections, indications of caesarean section, previous caesarean.

1. Department of Obstetrics \& Gynaecology, Lumbini Zonal Hospital, Butwal.

\author{
For Correspondence \\ Dr Shreedhar Acharya, MD \\ Obstetrics \& Gynaecology \\ Lumbini Zonal Hospital, Butwal \\ Phone+977-9857021374
}




\section{INTRODUCTION:}

It is estimated that 18.5 million Caesarean Sections (CS) are conducted annually worldwide and about one-third of them are done without medical indications and described as "unnecessary". Although developed countries account for most of the rise in the trend of unnecessary CS, more studies report a similar trend in developing countries, putting a strain on existing but limited healthcare resources, jeopardizing family's financial security and presenting a barrier to equitable universal coverage.

There has been a remarkable increase in the rate of caesarean section (CS) in both developed and developing countries in the past decades, increasing from about $5 \%$ in developed countries in the early 1970 s to more than $50 \%$ in some regions of the world in the late $1990 \mathrm{~s}^{1-4}$. Based on a survey by the World Health Organization (WHO) on methods of delivery during the period 20072008, the rates of CS in China and other Asian countries were $46 \%$ and $27 \%$, respectively ${ }^{5}$, despite the fact that in 1985 , WHO recommended that no region should have a CS rate over $10-15 \%{ }^{6,7}$.

Caesarean section rate below $5 \%$ seems to be associated with the gaps in obstetric care leading to poor health outcomes for mother and child whereas rates over $15 \%$ do not seem to improve either maternal or fetal health ${ }^{8}$. So this study was done regarding the indications for caesarean sections at our Government zonal level hospital to find out the commonest indications of caesarean section, so as to curtail the increasing number of caesarean delivery.

\section{MATERIAL \& METHODS}

This was a retrospective review of case notes of 1367 women who had caesarean delivery for various indications at Lumbini zonal hospital, Butwal over the period of one year from $2071 / 4 / 1$ to $2072 / 3 / 32\left(17^{\text {th }}\right.$ July 2014 to $16^{\text {th }}$ July2015). The case files of all the women, who had caesarean delivery over the above period, were reviewed and analysed for patients age, parity, incidence, indications and type of caesarean sections. The ethical approval was taken from the hospital administration and data were collected. When caesarean section was performed as a preplanned operation to ensure the best quality anaesthesia, neonatal resuscitation and nursing care services, it was classified as elective caesarean section and when the operation was performed due to unforeseen or acute obstetric emergencies, it was labelled as emergency caesarean section. Caesarean section done for the first time on women was labelled as primary CS and if it was performed with history of one or more previous caesarean section, it was labelled Repeat
CS. All the data was entered in MS Excel and analysed using SPSS programme.

\section{RESULTS}

There were 7589 deliveries during the one year period from $17^{\text {th }}$ July 2014 to $16^{\text {th }}$ July 2015 at Lumbini Zonal Hospital, Butwal. The incidence of caesarean section at Lumbini Zonal Hospital, Butwal for the year was $17.34 \%$ (Table 1). The commonest recorded indications for CS were: previous CS (275) (20.89\%), non progress of labour (245), cephalo-pelvic disproportion (222), failed induction (215), malpresentation (109), fetal distress (70), severe oligo with unfavourable cervix 95, gestational hypertension and eclampsia (60)and placenta previa and antepartum haemorrhage (25). The majority of $550 \mathrm{CS}$ were performed as emergency and rest were elective. Previous CS, "post-term pregnancy with unengaged head labelling as CPD and malpresentations were common indications for elective CS with "post dates with failed induction" the commonest indication for CS in primi parous women.

\begin{tabular}{|l|l|l|}
\hline Mode of delivery & Number of delivery & Rate \% \\
\hline Vaginal & 6273 & 82.65 \\
\hline Caesarean & 1316 & 17.34 \\
\hline Delivery total & 7589 & \\
\hline
\end{tabular}

Table 1. Caesarean section rate

In this study caesarean sections were seen more common in primi para than multiparous women (Table 2)

\begin{tabular}{|l|l|l|}
\hline Parity & Total cases & Percentage \\
\hline Primipara & 702 & 53.34 \\
\hline Multipara & 614 & 46.65 \\
\hline
\end{tabular}

Table 2. Showing caesarean section rate among primi para and multi para

If age of women having caesarean delivery is considered, it was significantly more common in the age group of 20-30 yrs $(93.16 \%)$ with rate of teenagers pregnancy $5.54 \%$ (Table 3 )

\begin{tabular}{|l|l|l|}
\hline Age in yrs & Total cases & Percentage \\
\hline$<20$ & 73 & 5.54 \\
\hline $20-30$ & 1226 & 93.16 \\
\hline$>30$ & 17 & 1.29 \\
\hline
\end{tabular}

Table 3. Showing caesarean section rate in different age groups. 
In this study, elective caesarean rate was higher than emergency CS. (Table 4)

\begin{tabular}{|l|l|l|}
\hline Type & Total cases & Percentage \\
\hline Elective & $\mathbf{7 6 6}$ & $\mathbf{5 8 . 2 0}$ \\
\hline Emergency & $\mathbf{5 5 0}$ & $\mathbf{4 1 . 7 9}$ \\
\hline
\end{tabular}

Table 4 showing comparison of elective versus emergency caesarean.

\section{DISCUSSION}

This study has documented the indications for both elective and emergency CS conducted at Lumbini Zonal Hospital, a government level hospital, at Butwal and also tried to explore how factors other than a real medical reason for CS influence decision-making by health care providers.

Previous CS was the leading indication (20.89\%) for CS in this study and was the main reason recorded for all elective $\mathrm{CS}$. We estimated that there were proportion of CS conducted without a clear medical indication in this study. This could be high if a more critical analysis of the indicators for CS were possible. For example, not all women who had a previous CS may have required a repeat $\mathrm{CS}$. CS for indications such as "post term dates", unfavorable cervix and rupture of membranes could perhaps have been avoided if adequate guidelines and resources are in place for safe induction of labor or augmentation. Other reasons for the "overmedicalization" of maternity care relate to a failure and inability to implement good quality evidence practices such as ensuring companionship during labor and delivery, a choice and range of drugs and methods to alleviate pain and assisted delivery where indicated using ventouse or forceps ${ }^{8}$.

A study of 300 cases of CS in Pakistan conducted at a tertiary hospital noted $11.3 \%$ of these were elective CS and $88.7 \%$ were performed as an emergency $\mathrm{CS}^{9}$. Sultana et al. reported on 209 CS performed at district level in Pakistan with 11.9\% done as an emergency and $82.4 \%$ as elective ${ }^{10}$. In Nepal at tertiary level, slow progress in labor, previous CS, fetal distress and breech presentation were the commonest indicators for $\mathrm{CS}^{11}$. Another study conducted in urban Bangladesh, reported fetal distress, pre-eclampsia and cervical dystocia as the commonest indications for $\mathrm{CS}^{12}$. In our study in contradiction, emergency caesareans only contributes $41.79 \%$ of all caesarean deliveries and a lot of caesareans were also done electively for various indications. In our study CPD comprised a huge percentage $(16.86 \%)$ of which the real medical indication was not so clear. On reviewing the chart note, the findings suggested to have term or post-term pregnancy with unengaged fetal head as well as relatively bigger size of baby clinically judged by clinicians. The patients didn't have trial of labour to diagnose as definite CPD . Similarly, this hospital has protocol to have elective repeat caesarean for the previous caesarean at 38-39 wks gestation which contributed repeat caesarean as major percentage of indication. vaginal birth after CS were only planned for cases who presented at advanced active stage of labour and for those who wanted to try for vaginal birth despite counselling for the protocol.

Other studies from low and middle income countries suggest the proportion of CS performed without a clear reported medical indication might be higher. Out of the 303 caesarean sections they reviewed in Tanzania, 25\% were based on "inappropriate" indications and in an additional $38 \%$ of cases the indication was not clear ${ }^{13}$.

A shortage of staff was given as the explanation for why most of the emergency CS, were conducted during daytime working hours as well as lots of elective caesareans were performed earlier to avoid emergency caesareans at off hours of government hospital. This is supported by the findings of Khan $\mathrm{R}$ et al. who reported that the unavailability of both an Obstetrician and Anaesthetist when needed is often a reason why emergency obstetric care services including CS are not available 24/7 in rural areas of Bangladesh ${ }^{14}$. This is likely also to be a potential reason for the increase in the number of elective CS performed. The inability to provide rapid emergency obstetric care (including CS) when this is needed outside of "normal working hours" is a real barrier to providing good quality, patient friendly, evidence based maternity care including CS for clear medical indication.

A range of factors other than adherence to evidence based guidelines and identification of a clear medical indication for a $\mathrm{CS}$ as mode of delivery influence providers' decision to conduct CS in public hospitals.

There was $53.34 \%$ off cases who were primipara having caesarean deliveries which also contributes a significant number of caesareans in their subsequent pregnancy as vaginal birth after caesareans is generally thought to be a less possible event due to fear of dehiscence and rupture during trial of labour.

A significant number of elective as well as emergency caesareans were also accumulated at this referral hospital as we have facility for operative delivery free of cost under government safe maternity and neonatal health programme as 
well as free neonatal care and treatment facilities. In addition women will get travel expense also to encourage institutional delivery with the aim of reducing maternal morbidity and mortality.

\section{CONCLUSION}

In this study, caesarean section rate is $17.34 \%$, which is slightly higher than WHO guideline (5-15\%). Performing a CS more frequently for cases where this is no real indication constitutes an unnecessary strain on existing and already limited resources whereas benefits of indicated caesarean delivery cannot be denied .Previous CS was the most common indication followed by nonprogress of labour, cephalopelvic disproportion, and failed induction. Since, the previous CS is a common indication, trial of labour should be encouraged in properly selected cases with adequate counselling so that reduction in overall caesarean section rate can be met with promotion of trial of labour. Avoiding "unnecessary" CS would perhaps free resources and could contribute to making $\mathrm{CS}$ accessible to those who really need this intervention as part of the emergency obstetric and newborn care package that needs to be available $24 / 7$ to manage potentially life threatening complications in both women and newborn babies.

\section{Limitations and further study}

One of the limitations of this study is its focus on public government hospital. This provides good information regarding what happens in public hospitals at district levels but we are aware that a substantial number of CS are increasingly being performed at private hospitals even in less urban settings. Factors influencing decision making for CS reported in this study only reflect the views of healthcare providers. Patients' views that would have highlighted other important aspects were not explored.

A recent Cochrane review highlighted that there is emerging evidence that guideline implementation, a mandatory second opinion and peer-review feedback could all lead to a reduction in $\mathrm{CS}$ cases $^{3}$.

\section{REFERENCES}

1. Schmidt S: Use and abuse of cesarean section - a transatlantic evaluation. JPerinat Med 2009, 37:565-568.

2. Lavender T, Hofmeyr GJ, Neilson JP, Kingdon C, Gyte GM: Caesarean section for non-medical reasons at term. Cochrane Database Syst Rev 2012., 3
3. Khunpradit S, Tavender E, Lumbiganon P, Laopaiboon M, Wasiak J, Gruen RL: Non-clinical interventions for reducing unnecessary caesarean section. Cochrane Database Syst Rev 2011, 6

4. World Health Organization: The global numbers and costs of additionally needed and unnecessary caesarean sections performed per year: overuse as a barrier to universal coverage. 2012

5. World Bank: World Development Indicators Bangladesh. 2013.

6. UNFPA: Bangladesh Maternal Mortality and Healthcare Survey. Published 2010

7. Measure DHS: Bangladesh Demographic and Health Survey. Published 2011.

8. Lewis J. London; Thousand Oaks, Calif: Sage Publications; 2003:219-262.

9. Khawaja NP, Yousaf T, Tayyeb R: Analysis of caesarean delivery at a tertiary care hospital in Pakistan. J Obstet Gynaec 2004, 24:139-141

10. Sultana A, un Nisa A: Indications of caesarean section in a district head quarter hospital for women. J Ayub Med Coll 2003, 15:36-38.

11. Khanal R: Caesarean delivery at Nepal Medical College Teaching Hospital, Kathmandu, Nepal. Nepal Med Coll J 2004, 6:53-55.

12. Saha L, Chowdhury SB: Study on primary cesarean section. Mymensingh MedJ2011, 20:292-297.

13. Maaleo N, Bygbjerg IC, Sorensen BL, Onesmo R, Secher NJ; Prolonged labour as indication for emergency caesarean section; a quality assurance analysis by crierion-based audit at at two Tanzanian hospitals, Brit J Obstet Gynaec, 2012, 119:605-613

14. Khan R, Blum LS, Sultana M, Bilkis S, Koblinsky M: An examination of women experiencing obstetric complications requiring emergency care: perceptions and sociocultural consequences of caesarean sections in Bangladesh. J Health PopulNutr 2012, 30:159-171. 